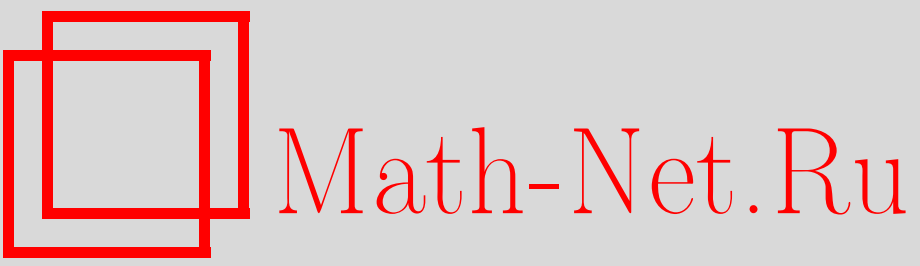

В. А. Москаленко, Теория возмущений для периодической модели Андерсона. II. Сверхпроводящее состояние, ТМФ, 1998, том 116, номер 3, 456-473

DOI: https://doi.org/10.4213/tmf916

Использование Общероссийского математического портала Math-Net.Ru подразумевает, что вы прочитали и согласны с пользовательским соглашением

http://www . mathnet.ru/rus/agreement

Параметры загрузки:

IP : 54.197 .130 .99

26 апреля 2023 г., 13:43:48 
ТЕОРЕТИЧЕСКАЯ

И МАТЕМАТИЧЕСКАЯ

ФИЗИКА

Том 116, № 3

сентябрь, 1998

(C) 1998 г.

В.А. Москаленко*

\title{
ТЕОРИЯ ВОЗМУШЕНИЙ ДЛЯ ПЕРИОДИЧЕСКОЙ МОДЕЛИ АНДЕРСОНА. II. СВЕРХПРОВОДЯЩЕЕ СОСТОЯНИЕ
}

\begin{abstract}
Новая диаграммная техника, развитая для сильно коррелированных электронных систем, используется при исследовании свойств периодической модели Андерсона в сверхпроводящем состоянии. Для равноправного описания как нормальных, так и аномальных функций Грина вводится дополнительное зарядовое квантовое число, которое отличает операторы рождения электронов от операторов уничтожения. Выведены уравнения Дайсона для функций Грина зонных и локализованных электронов при наличии сверхпроводимости. Полученные уравнения допускают существование как синглетной, так и триплетной сверхпроводимости. В случае синглетной сверхпроводимости установлено соответствие полученных уравнений и спинорного формализма Горькова-Намбу.
\end{abstract}

\section{1. ВВЕДЕНИЕ}

Исследование свойств систем с сильными электронными корреляциями является центральной задачей квантовой теории систем многих частиц, теории твердого тела и квантовой химии. В самом деле, в теории систем с тяжелыми фермионами и высокотемпературной сверхпроводимостью, а также в теории поверхностной абсорбции и др. локализованные электроны испытывают сильное кулоновское отталкивание. Это взаимодействие должно быть учтено как основной фактор, определяющий свойства этих систем, и поэтому не может рассматриваться как возмушение. Как следствие этого положения возникает необходимость развития теоретических методов исследования, выходящих за рамки квантово-полевой теории со слабыми взаимодействиями.

Некоторые из вышеперечисленных систем могут пребьвать как в диэлектрическом, так и в нормальном металлическом или сверхпроводящем состоянии. Поэтому теория сильно коррелированных электронных систем должна быть развита с учетом возможного нарушения свойств симметрии системы. Именно нарушение симметрии системы, связанное с законом сохранения числа частиц, и возникновение сверхпроводящего состояния будут исследоваться в дальнейшем.

* Институт прикладной физики АН Молдовы, Кишинев, Молдова 
В настоящее время наиболее известны две модели квантовых систем с сильным кулоновским отталкиванием электронов: модель Хаббарда [1] и модель Андерсона [2], для которых были предложены многочисленные квантово-полевые и численные методы исследования. Здесь мы не будем останавливаться на наиболее сушественных этапах развития теории указанных моделей, полагая, что в наших работах [3-9] по исследованию модели Хаббарда и в статьях [10-12], посвященных модели Андерсона, можно найти необходимые ссылки.

Цель данной работы состоит в обобщении новой диаграммной техники, развитой для нормальной модели Андерсона и изложенной в работах [10-12], на случай существования в данной модели сверхпроводящей фазы. Для этого, как известно, следует ввести квазисредние Боголюбова [13] для связанных куперовских пар электронов или аномальные функции Грина-Горькова [14].

Предложенный в данной статье метод исследования обобщает теорию сверхпроводимости модели Хаббарда, развитую в работах $[8,9]$, и представляет собой непосредственное обобшение на случай сверхпроводимости результатов работы [11]. Это обобшение должно учитывать тот факт, что в модели Андерсона, в отличие от однозонной модели Хаббарда, имеются две группы с- и f-электронов, состояния которых гибридизированы.

Модель Андерсона предполагается невырожденной, а гибридизация - локальной. Гамильтониан этой модели имеет вид

$$
H=H_{\mathrm{c}}^{0}+H_{\mathrm{f}}^{0}+H_{\mathrm{int}}
$$

где

$$
\begin{aligned}
H_{\mathrm{c}}^{0} & =\sum_{\vec{k} \sigma}(\epsilon(\vec{k})-\mu) c_{\vec{k} \sigma}^{+} c_{\vec{k} \sigma}=\sum_{\vec{k} \sigma} H_{\mathrm{c} \sigma}^{0}(\vec{k}), \\
H_{\mathrm{f}}^{0} & =\sum_{i} H_{\mathrm{f}}^{0}(i)=\sum_{i \sigma}\left(E_{\mathrm{f}}-\mu\right) f_{i \sigma}^{+} f_{i \sigma}+U \sum_{i} n_{i \uparrow}^{\mathrm{f}} n_{i \downarrow}^{\mathrm{f}}, \\
H_{\mathrm{int}} & =V \sum_{i \sigma}\left(f_{i \sigma}^{+} c_{i \sigma}+c_{i \sigma}^{+} f_{i \sigma}\right), \\
c_{i \sigma} & =\frac{1}{\sqrt{N}} \sum_{\vec{k}} c_{\vec{k} \sigma} e^{-i \vec{k} \vec{R}_{i}}, \quad n_{i \sigma}^{\mathrm{f}}=f_{i \sigma}^{+} f_{i \sigma} .
\end{aligned}
$$

Здесь операторы $c_{\vec{k} \sigma}, c_{\vec{k} \sigma}^{+}$относятся к электронам проводимости, обладаюшим волновым вектором $\vec{k}$ и спином $\sigma$ и принадлежашим к энергетической зоне $\epsilon(k)$. Операторы $f_{i \sigma}, f_{i \sigma}^{+}$относятся к локализованным в узлах решетки электронам $4 \mathrm{f}$ - или $5 \mathrm{f}$-атомных оболочек с энергией $E_{\mathrm{f}}$.

Между f-электронами действует сильное кулоновское отталкивание $U$, которое является основным слагаемым гамильтониана; $\mu$ - химический потенциал системы. Так как с-электроны могут принадлежать не только к s-, но и к d-оболочкам атомов, то учет их кулоновского отталкивания также может быть актуальным. Здесь для простоты корреляции с-электронов не рассматриваются. Из-за сильной локализованности f-электронов они не могут перескакивать с узла на узел решетки и их делокализация 
связана только с гибридизацией $\mathrm{f}-$ и с-состояний с матричным элементом $V$. Очевидно, что при $V \neq 0$ сверхпроводимость в данной модели с двумя подсистемами наступает одновременно в обеих подсистемах.

В этой работе развита термодинамическая теория возмушений для системы с гамильтонианом (1), находяшейся в сверхпроводяшем состоянии, в предположении, что появление слагаемого вследствие гибридизации с- и f-электронов является возмущением состояния двух подсистем.

Гамильтониан с-подсистемы $H_{\mathrm{c}}^{0}$ диагонален в зонном представлении, тогда как гамильтониан $H_{\mathrm{f}}^{0}$ диагонализуется с помощью операторов Хаббарда $[1,11]$. Поэтому в нулевом приближении теории возмушений статистический оператор большого канонического ансамбля системы факторируется в импульсном представлении для с- и в локальном представлении для f-электронов:

$$
e^{-\beta H^{0}}=\prod_{\vec{k} \sigma} e^{-\beta H_{\mathrm{c} \sigma}^{0}(\vec{k})} \prod_{i} e^{-\beta H_{\mathrm{f}}^{0}(i)} .
$$

Термодинамические ряды теории возмушений для динамических величин системы основаны на разложении в ряд оператора эволюции

$$
\begin{aligned}
U(\beta) & =\exp \left(-\int_{0}^{\beta} H_{\mathrm{int}}(\tau) d \tau\right)= \\
& =\sum_{n=0}^{\infty} \frac{(-1)^{n}}{n !} \int_{0}^{\beta} d \tau_{1} \ldots \int_{0}^{\beta} d \tau_{n} T\left(H_{\mathrm{int}}\left(\tau_{1}\right) \ldots H_{\mathrm{int}}\left(\tau_{n}\right)\right)
\end{aligned}
$$

и на последующем статистическом усреднении хронологических произведений операторов с матрицей плотности (3). Это усреднение обозначается через $\langle\ldots\rangle_{0}$.

\section{2. ОДНОЧАСТИЧНЫЕ ФУНКЦИИ ГРИНА СВЕРХПРОВОДЯЩЕЙ ФАЗЫ}

В этом разделе развита диаграммная техника для вычисления полных температурных функций Грина с- и f-электронов в сверхпроводящем состоянии системы.

Для с-подсистемы эти величины имеют вид

$$
\begin{aligned}
& G^{\mathrm{c}}\left(x \mid x^{\prime}\right)=-\left\langle T c(x) \bar{c}\left(x^{\prime}\right) U(\beta)\right\rangle_{0}^{\mathrm{c}}, \\
& F^{\mathrm{c}}\left(x \mid x^{\prime}\right)=-\left\langle T c(x) c\left(x^{\prime}\right) U(\beta)\right\rangle_{0}^{\mathrm{c}}, \\
& \bar{F}^{\mathrm{c}}\left(x \mid x^{\prime}\right)=-\left\langle T \bar{c}(x) \bar{c}\left(x^{\prime}\right) U(\beta)\right\rangle_{0}^{\mathrm{c}},
\end{aligned}
$$

где операторы с-электронов записаны в представлении взаимодействия

$$
\begin{aligned}
& c(x)=e^{\tau H^{0}} c(\vec{x}, \sigma) e^{-\tau H^{0}}, \\
& \bar{c}(x)=e^{\tau H^{0}} c^{+}(\vec{x}, \sigma) e^{-\tau H^{0}} .
\end{aligned}
$$


Параметр $x$ обозначает совокупность $(\vec{x}, \sigma, \tau)$, и верхний индекс с у средних статистических указывает на связность диаграмм, которые следует учитывать в правой части (5).

Для f-подсистемы аналогичные функции определяются заменой с-операторов на fоператоры. Функции Грина этой подсистемы снабжены индексом f.

Благодаря смешиванию с- и f-электронов возникают также смешанные функции Грина вида

$$
\begin{aligned}
& G^{\mathrm{cf}}\left(x \mid x^{\prime}\right)=-\left\langle T c(x) \bar{f}\left(x^{\prime}\right) U(\beta)\right\rangle_{0}^{\mathrm{c}}, \\
& F^{\mathrm{cf}}\left(x \mid x^{\prime}\right)=-\left\langle T c(x) f\left(x^{\prime}\right) U(\beta)\right\rangle_{0}^{\mathrm{c}}, \\
& \bar{F}^{\mathrm{cf}}\left(x \mid x^{\prime}\right)=-\left\langle T \bar{c}(x) \bar{f}\left(x^{\prime}\right) U(\beta)\right\rangle_{0}^{\mathrm{c}} .
\end{aligned}
$$

Имеются, очевидно, также функции $G^{\mathrm{f} \mathrm{c}}, F^{\mathrm{f} \mathrm{c}}$ и $\bar{F}^{\mathrm{f} \mathrm{c}}$, которые получаются из вышеприведенных функций перестановкой с- и f-операторов между собой. Функции $G$ существуют как в нормальном, так и в сверхпроводящем состояниях, тогда как функции $F$ и $\bar{F}$ только в сверхпроводящем состоянии.

В нулевом приближении по гибридизации эти последние функции равны нулю. Полные функции $F$ и $\bar{F}$ отличаются от нуля из-за нарушения симметрии и гибридизации.

Подстановка в формулы (5) и (7) оператора эволюции (4) приводит к необходимости вычислять термодинамические средние от хронологических произведений электронных операторов с- и f-подсистем. Вследствие факторизации матрицы плотности (3) эти вычисления выполняются независимо для с-подсистемы с помошюю теоремы Вика квантовой теории поля и независимо для f-подсистемы. Для f-подсистемы используется обобщенная теорема Вика, предложенная для сильно коррелированных систем в рабо$\operatorname{Tax}[3-7]$

Как уже отмечалось, в сверхпроводящем состоянии возможно появление отличных от нуля статистических средних от произведений операторов, в которых число операторов рождения не равно числу операторов уничтожения. Для достижения автоматизма в вычислении подобных средних целесообразно снабдить электронные операторы обеих подсистем дополнительным квантовым числом $\alpha$ со значениями \pm 1 по правилу

$$
c^{\alpha}(x)=\left\{\begin{array}{ll}
c(x) & \text { при } \alpha=1, \\
\bar{c}(x) & \text { при } \alpha=-1,
\end{array} \quad f^{\alpha}(x)= \begin{cases}f(x) & \text { при } \alpha=1, \\
\bar{f}(x) & \text { при } \alpha=-1 .\end{cases}\right.
$$

Это число вводит частицы и дырки и может быть названо зарядовым. В этом представлении оператор взаимодействия (2) принимает вид

$$
H_{\mathrm{int}}=V \sum_{i \sigma \alpha} \alpha f_{i \sigma}^{-\alpha} c_{i \sigma}^{\alpha}
$$

Очевидно, что введение нового квантового зарядового числа приводит к возникновению дополнительного суммирования по $\alpha$ во всех линиях диаграмм и к дополнительному множителю $\alpha$ в вершинах. При этом не нужно следить за тем, имеем мы дело с операторами рождения или уничтожения. Такую идентификацию можно делать после 
выяснения структуры уравнений динамических величин путем задания конкретных значений чисел $\alpha$.

В дальнейшем используются следующие определения одночастичных функций Грина:

$$
\begin{aligned}
& G_{\alpha \alpha^{\prime}}^{\mathrm{c}}\left(x \mid x^{\prime}\right)=-\left\langle T c^{\alpha}(x) c^{-\alpha^{\prime}}\left(x^{\prime}\right) U(\beta)\right\rangle_{0}^{\mathrm{c}}, \\
& G_{\alpha \alpha^{\prime}}^{\mathrm{f}}\left(x \mid x^{\prime}\right)=-\left\langle T f^{\alpha}(x) f^{-\alpha^{\prime}}\left(x^{\prime}\right) U(\beta)\right\rangle_{0}^{\mathrm{c}}, \\
& G_{\alpha \alpha^{\prime}}^{\mathrm{cf}}\left(x \mid x^{\prime}\right)=-\left\langle T c^{\alpha}(x) f^{-\alpha^{\prime}}\left(x^{\prime}\right) U(\beta)\right\rangle_{0}^{\mathrm{c}}, \\
& G_{\alpha \alpha^{\prime}}^{\mathrm{fc}}\left(x \mid x^{\prime}\right)=-\left\langle T f^{\alpha}(x) c^{-\alpha^{\prime}}\left(x^{\prime}\right) U(\beta)\right\rangle_{0}^{\mathrm{c}} .
\end{aligned}
$$

Вьполняются условия

$$
\begin{aligned}
& G_{\alpha \alpha^{\prime}}^{\mathrm{a}}\left(x \mid x^{\prime}\right)=-G_{-\alpha^{\prime},-\alpha}^{\mathrm{a}}\left(x^{\prime} \mid x\right), \quad \mathrm{a}=\mathrm{c}, \mathrm{f}, \\
& G_{\alpha \alpha^{\prime}}^{\mathrm{c} \mathrm{f}}\left(x \mid x^{\prime}\right)=-G_{-\alpha^{\prime},-\alpha}^{\mathrm{f} \mathrm{c}}\left(x^{\prime} \mid x\right) .
\end{aligned}
$$

Соотношение между определениями $(5),(7)$ и (10) одночастичных функций Грина очевидно $(\mathrm{a}=\mathrm{c}, \mathrm{f})$ :

$$
\begin{array}{rlrl}
G_{1,1}^{\mathrm{a}}\left(x \mid x^{\prime}\right) & =G^{\mathrm{a}}\left(x \mid x^{\prime}\right), & G_{1,-1}^{\mathrm{a}}\left(x \mid x^{\prime}\right) & =F^{\mathrm{a}}\left(x \mid x^{\prime}\right), \\
G_{-1,1}^{\mathrm{a}}\left(x \mid x^{\prime}\right) & =\bar{F}^{\mathrm{a}}\left(x \mid x^{\prime}\right), & \\
G_{-1,-1}^{\mathrm{a}}\left(x \mid x^{\prime}\right) & =-G^{\mathrm{a}}\left(x^{\prime} \mid x\right), \quad G_{-1,-1}^{\mathrm{cf}}\left(x \mid x^{\prime}\right)=-G^{\mathrm{fc}}\left(x^{\prime} \mid x\right) .
\end{array}
$$

Переход к нормальному состоянию системы налагает ограничения на возможные значения зарядовых квантовых чисел термодинамических средних, а именно, для каждого такого среднего сумма всех чисел $\alpha$ должна равняться нулю. Из определений (10) видно, что условие $\alpha-\alpha^{\prime}=0$ выполняется только для функций Грина нормального состояния. При этом ограничении остаются две возможности: $\alpha=\alpha^{\prime}=1$ и $\alpha=\alpha^{\prime}=-1$. Обе они приводят при суммировании по $\alpha$ к удвоению коэффициента диаграммы по сравнению со значением в сверхпроводяшем состоянии. Это обстоятельство объясняет различие числа эквивалентных диаграмм и коэффициентов при них в нормальном состоянии [11] и в сверхпроводящем состоянии, которое обсуждается ниже.

Дальнейшее исследование свойств сверхпроводяшей фазы основано на определениях (9), (10) и выражении (4) для оператора эволюции.

Теорема Вика для сверхпроводяшего состояния некоррелированных с-электронов хорошо известна и приводится лишь для сравнения с обобшенной теоремой Вика. Она имеет вид

$$
\left\langle T c^{\alpha_{1}}\left(x_{1}\right) \ldots c^{\alpha_{2 n}}\left(x_{2 n}\right)\right\rangle_{0}=\sum_{P}(-1)^{P} P G_{\alpha_{1} \alpha_{2}}^{\mathrm{c}(0)}\left(x_{1} \mid x_{2}\right) \ldots G_{\alpha_{2 n-1} \alpha_{2 n}}^{\mathrm{c}(0)}\left(x_{2 n-1} \mid x_{2 n}\right)
$$

Правая часть (12) содержит $(2 n-1)$ !! членов, где $P$ - знак операции перестановки индексов с-операторов. Здесь индексом (0) отмечены функции Грина нулевого приближения теории возмушения. Как уже отмечалось выше, для нормальной фазы должны иметь 
место равенства $\alpha_{1}+\cdots+\alpha_{2 n}=0$ и число членов правой части (12) становиться равным $n$ !.

Наличие дополнительного зарядового квантового числа $\alpha$ не влияет на формулировку теоремы Вика, т.к. для ее реализации сушественны остальные квантовые числа.

При учете сильных корреляций, присуших f-электронам, теорему (12) следует обобщить. Обобщенная теорема Вика содержит дополнительные по сравнению с правой частью (12) слагаемые, содержашие новые элементы теории, а именно неприводимые одноузельные многочастичные функции Грина f-электронов

$$
\begin{gathered}
G_{n}^{(0) \operatorname{ir}}\left[\alpha_{1}, x_{1} ; \ldots ; \alpha_{2 n}, x_{2 n}\right]=\left\langle T f^{\alpha_{1}}\left(x_{1}\right) \ldots f^{\alpha_{2 n}}\left(x_{2 n}\right)\right\rangle_{0}^{\mathrm{ir}}= \\
=\delta \vec{x}_{1}, \vec{x}_{2} \ldots \delta \vec{x}_{1}, \vec{x}_{2 n}\left\langle T f_{\sigma_{1}}^{\alpha_{1}}\left(\tau_{1}\right) \ldots f_{\sigma_{2 n}}^{\alpha_{2 n}}\left(\tau_{2 n}\right)\right\rangle_{0}^{\mathrm{ir}} .
\end{gathered}
$$

Последний множитель правой части (13) строится по правилу образования кумулянт Кубо, т.е. путем вычитания из $n$-частичной локальной функции

$$
\left\langle T f_{\sigma_{1}}^{\alpha_{1}}\left(\tau_{1}\right) \ldots f_{\sigma_{2 n}}^{\alpha_{2 n}}\left(\tau_{2 n}\right)\right\rangle_{0}
$$

всех возможных произведений функций Грина меньшего числа частиц $n_{1}, n_{2} \ldots$ при условии, что $n_{1}+n_{2}+\cdots=n$.

Простейшая, т.е. двухчастичная, функция Грина имеет вид

$$
\begin{aligned}
& \left\langle T f_{\sigma_{1}}^{\alpha_{1}}\left(\tau_{1}\right) f_{\sigma_{2}}^{\alpha_{2}}\left(\tau_{2}\right) f_{\sigma_{3}}^{\alpha_{3}}\left(\tau_{3}\right) f_{\sigma_{4}}^{\alpha_{4}}\left(\tau_{4}\right)\right\rangle_{0}^{\mathrm{ir}}=\left\langle T f_{\sigma_{1}}^{\alpha_{1}}\left(\tau_{1}\right) f_{\sigma_{2}}^{\alpha_{2}}\left(\tau_{2}\right) f_{\sigma_{3}}^{\alpha_{3}}\left(\tau_{3}\right) f_{\sigma_{4}}^{\alpha_{4}}\left(\tau_{4}\right)\right\rangle_{0}- \\
& -\left\langle T f_{\sigma_{1}}^{\alpha_{1}}\left(\tau_{1}\right) f_{\sigma_{2}}^{\alpha_{2}}\left(\tau_{2}\right)\right\rangle_{0}\left\langle T f_{\sigma_{3}}^{\alpha_{3}}\left(\tau_{3}\right) f_{\sigma_{4}}^{\alpha_{4}}\left(\tau_{4}\right)\right\rangle_{0}+ \\
& +\left\langle T f_{\sigma_{1}}^{\alpha_{1}}\left(\tau_{1}\right) f_{\sigma_{3}}^{\alpha_{3}}\left(\tau_{3}\right)\right\rangle_{0}\left\langle T f_{\sigma_{2}}^{\alpha_{2}}\left(\tau_{2}\right) f_{\sigma_{4}}^{\alpha_{4}}\left(\tau_{4}\right)\right\rangle_{0}- \\
& -\left\langle T f_{\sigma_{1}}^{\alpha_{1}}\left(\tau_{1}\right) f_{\sigma_{4}}^{\alpha_{4}}\left(\tau_{4}\right)\right\rangle_{0}\left\langle T f_{\sigma_{2}}^{\alpha_{2}}\left(\tau_{2}\right) f_{\sigma_{3}}^{\alpha_{3}}\left(\tau_{3}\right)\right\rangle_{0} .
\end{aligned}
$$

До тех пор, пока источник связанных электронных пар, нарушающий закон сохранения числа частищ системы, не устремлен к нулю, сумма зарядовых индексов $\alpha_{1}+\cdots+\alpha_{2 n}$ неприводимых функций Грина может принимать любые четные значения от $-2 n$ до $+2 n$. Детали определения неприводимых функций Грина можно найти в работах $[3,11]$.

После введения новых элементов теории сформулируем обобшенную теорему Вика для f-электронов:

$$
\begin{aligned}
& \left\langle T f^{\alpha_{1}}\left(x_{1}\right) \ldots f^{\alpha_{2 n}}\left(x_{2 n}\right)\right\rangle_{0}= \\
& =\sum_{P}(-1)^{P} P G_{\alpha_{1},-\alpha_{2}}^{\mathrm{f}(0)}\left(x_{1} \mid x_{2}\right) \ldots G_{\alpha_{2 n-1},-\alpha_{2 n}}^{\mathrm{f}(0)}\left(x_{2 n-1} \mid x_{2 n}\right)+ \\
& \quad+\sum_{P}(-1)^{P} P \sum_{\substack{n_{1}, \ldots, n_{p} \\
n_{1}+\cdots+n_{p}=n}} G_{n_{1}}^{(0) \operatorname{ir}}\left[\alpha_{1}, x_{1} ; \ldots ; \alpha_{2 n_{1}}, x_{2 n_{1}}\right] \times \cdots \\
& \quad \ldots \times G_{n_{p}}^{(0) \operatorname{ir}}\left[\alpha_{2 n-2 n_{p}+1}, x_{2 n-2 n_{p}+1} ; \ldots ; \alpha_{2 n}, x_{2 n}\right]+ \\
& \quad+G_{n}^{(0) \operatorname{ir}}\left[\alpha_{1}, x_{1} ; \ldots ; \alpha_{2 n}, x_{2 n}\right] .
\end{aligned}
$$




$$
\left\langle\left(U^{(4)}\right\rangle_{0}^{c}=-\frac{v^{2}}{2}\right.
$$
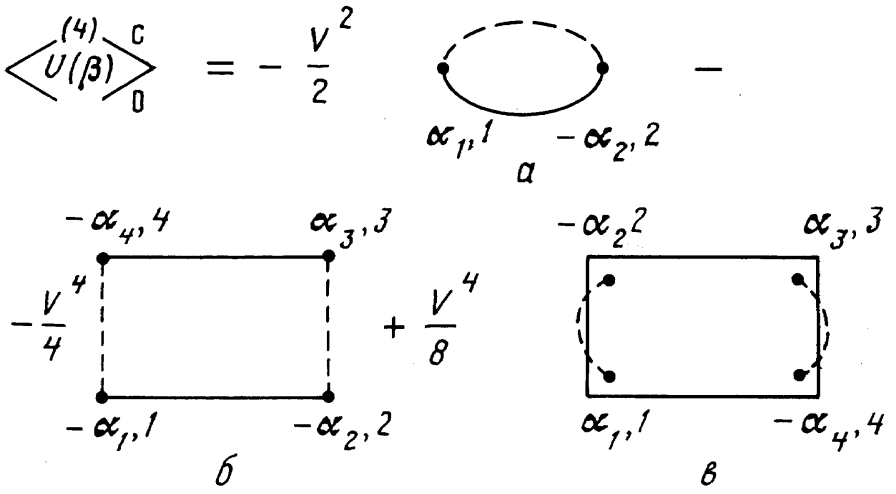

Рис. 1

Записанная в таком виде обобщенная теорема Вика использует только алгебраические свойства фермиевских операторов и в этом смысле сушественно отличается от обобшенной теоремы Вика для операторов Хаббарда работ [15-17]. В нашем подходе необходимость использования операторов Хаббарда проявляется на этапе вычисления одночастичных и многочастичных функций Грина f-электронов. Форма (15) обобщенной теоремы Вика максимально приближена к теореме Вика в теории слабой связи. При равенстве нулю кулоновского взаимодействия все неприводимые функции Грина тождественно равны нулю и в правой части (15) сохраняется только первое слагаемое.

Приведем простейшие примеры использования сформулированных теорем Вика для c- и f-электронов при определении вкладов в термодинамический потенциал

$$
F=-\frac{1}{\beta}\langle U(\beta)\rangle_{0}^{\mathrm{c}}
$$

и ренормированные одночастичные функции Грина (10) системы. В (16) индекс с указывает на связность "вакуумных" диаграмм термодинамической теории возмушений.

В первых двух неисчезаюших порядках теории возмушений (во втором и четвертом) по параметру гибридизации $V$ получаем, что

$$
\begin{aligned}
\left\langle U^{(4)}(\beta)\right\rangle_{0}^{\mathrm{c}}= & -\frac{V^{2}}{2} \sum_{\alpha_{1} \alpha_{2}} \sum_{1,2} G_{\alpha_{1} \alpha_{2}}^{\mathrm{f}(0)}(1 \mid 2) \alpha_{2} G_{\alpha_{2} \alpha_{1}}^{\mathrm{c}(0)}(2 \mid 1) \alpha_{1}- \\
& -\frac{V^{4}}{4} \sum_{\alpha_{1}, \ldots, \alpha_{4}} \sum_{1, \ldots, 4} \alpha_{1} G_{\alpha_{1} \alpha_{2}}^{\mathrm{f}(0)}(1 \mid 2) \alpha_{2} G_{\alpha_{2} \alpha_{3}}^{\mathrm{c}(0)}(2 \mid 3) \alpha_{3} \times \\
& \times G_{\alpha_{3} \alpha_{4}}^{\mathrm{f}(0)}(3 \mid 4) \alpha_{4} G_{\alpha_{4} \alpha_{1}}^{\mathrm{c}(0)}(4 \mid 1)+ \\
& +\frac{V^{4}}{8} \sum_{\alpha_{1}, \ldots, \alpha_{4}} \sum_{1, \ldots, 4}\left\langle T f^{\alpha_{1}}(1) f^{-\alpha_{2}}(2) f^{\alpha_{3}}(3) f^{-\alpha_{4}}(4)\right\rangle_{0}^{\mathrm{ir}} \times \\
& \times G_{\alpha_{2} \alpha_{1}}^{\mathrm{c}(0)}(2 \mid 1) G_{\alpha_{4} \alpha_{3}}^{\mathrm{c}(0)}(4 \mid 3) \alpha_{1} \alpha_{2} \alpha_{3} \alpha_{4} .
\end{aligned}
$$




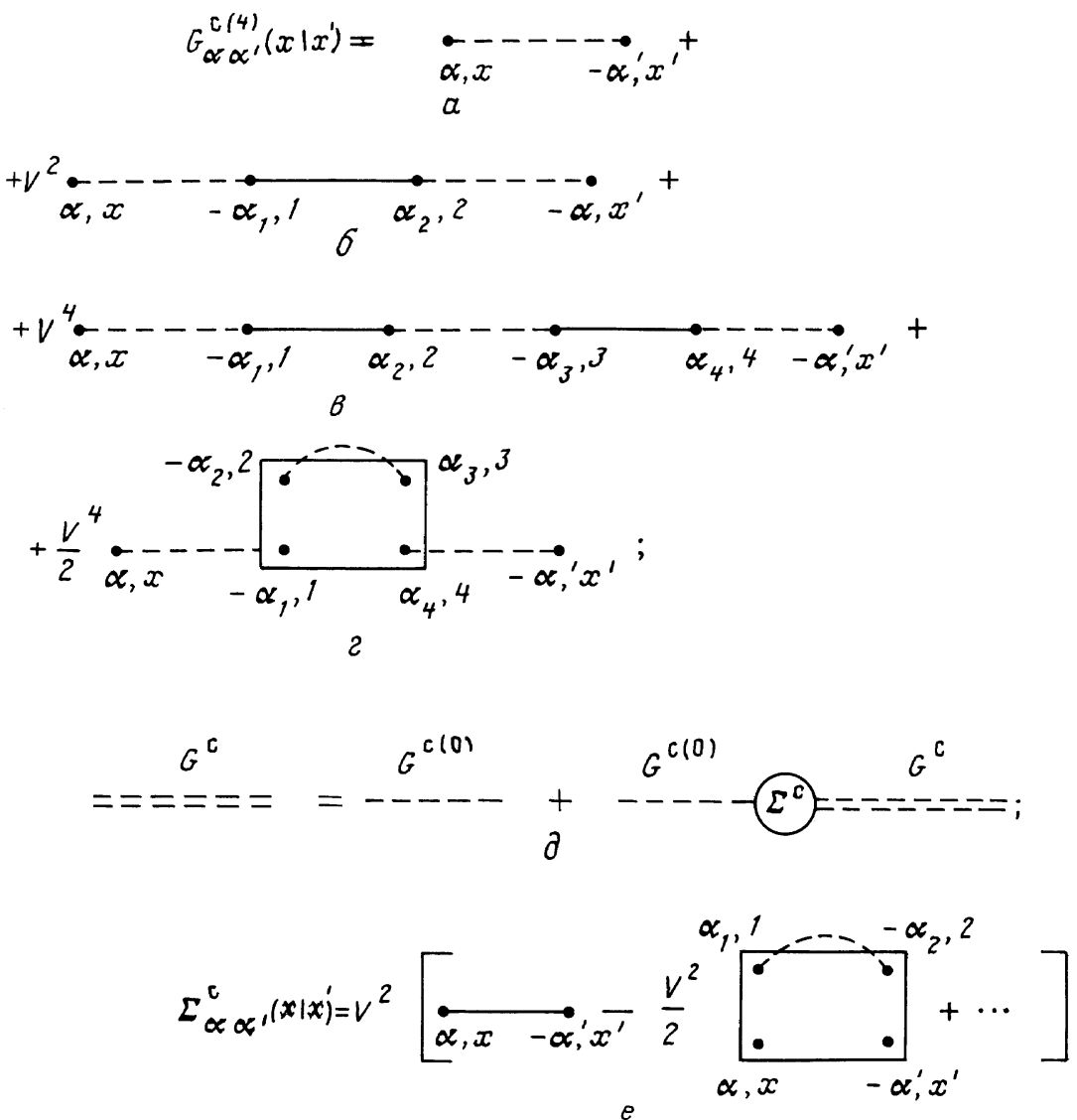

Рис. 2

Здесь под индексом 1 подразумевается совокупность $\left(\overrightarrow{1}, \sigma_{1}, \tau_{1}\right)$, и суммирование по этому индексу предполагает два суммирования по дискретным индексам $\overrightarrow{1}$ и $\sigma_{1}$ и одно интегрирование по $\tau_{1}$ в интервале $(0, \beta)$. Графическое изображение этих вкладов приведено на рис. 1, где представлены связанные вакуумные диаграммы второго ("a") и четвертого ( “б” и "в") порядков по константе гибридизации. Диаграммы "а" и “б” являются цепочечными, тогда как диаграмма “в” содержит простейшую неприводимую функцию Грина $G_{2}^{(0) \text { ir }}$; для простоты указаны только $\alpha$-числа f-операторов.

Сопоставление аналитического вклада (17) с диаграммами рис. 1 объясняет правила их построения. Отметим, что сплошным линиям, соединяющим две вершины, соответствуют одночастичные функции Грина нулевого приближения f-подсистемы, а штриховым линиям - аналогичная величина с-подсистемы. Каждая вершина диаграммы - это место, где действуют операторы $\mathrm{f}^{-\alpha}$ и с ${ }^{\alpha}$ с противоположными зарядовыми индексами. Прямоугольник соответствует неприводимой функции Грина f-электронов.

В нормальной фазе данной модели, исследованной в работе [11], все линии имеют заданные направления, а все узлы - направленные стрелки, указываюшие, что опера- 
тор уничтожается, если стрелка входит в узел, или рождается, если выходит из него. В сверхпроводящем случае число $\alpha$ может быть любым, т.к. в процессе образования операторных связок могут участвовать все возможные комбинации операторов рождения и уничтожения. Поэтому направление линий и вершин не указаны в данной статье. По этой же причине прямоугольники, окружающие $2 n$ вершин и изображающие $n$-частичную неприводимую функцию Грина, не содержат стрелок, входящих или выходяших из этих вершин. Все вакуумные диаграммы лишены внешних линий. Последние присутствуют во вкладах одночастичных или многочастичных функций Грина.

Так, например, для одночастичной функции Грина с-электронов с точностью до четвертого порядка по гибридизации получаем

$$
\begin{aligned}
G_{\alpha \alpha^{\prime}}^{\mathrm{c}(4)}\left(x \mid x^{\prime}\right)= & G_{\alpha \alpha^{\prime}}^{\mathrm{c}(0)}\left(x \mid x^{\prime}\right)+V^{2} \sum_{\alpha_{1} \alpha_{2}} \sum_{12} G_{\alpha \alpha_{1}}^{\mathrm{c}(0)}(x \mid 1) \alpha_{1} G_{\alpha_{1} \alpha_{2}}^{\mathrm{f}(0)}(1 \mid 2) \alpha_{2} G_{\alpha_{2} \alpha^{\prime}}^{\mathrm{c}(0)}\left(2 \mid x^{\prime}\right)+ \\
& +V^{4} \sum_{1, \ldots, 4 \alpha_{1}, \ldots, \alpha_{4}} G_{\alpha \alpha_{1}}^{\mathrm{c}(0)}(x \mid 1) \alpha_{1} G_{\alpha_{1} \alpha_{2}}^{\mathrm{f}(0)}(1 \mid 2) \alpha_{2} G_{\alpha_{2} \alpha_{3}}^{\mathrm{c}(0)}(2 \mid 3) \alpha_{3} \times \\
& \times G_{\alpha_{3} \alpha_{4}}^{\mathrm{f}(0)}(3 \mid 4) \alpha_{4} G_{\alpha_{4} \alpha^{\prime}}^{\mathrm{c}(0)}\left(4 \mid x^{\prime}\right)+ \\
& +\frac{V^{4}}{2} \sum_{\alpha_{1}, \ldots, \alpha_{4} 1, \ldots, 4} \sum_{\alpha \alpha_{1}} G^{\mathrm{c}(0)}(x \mid 1) \alpha_{1}\left\langle T f^{\alpha_{1}}(1) f^{-\alpha_{2}}(2) f^{\alpha_{3}}(3) f^{-\alpha_{4}}(4)\right\rangle_{0}^{\mathrm{ir}} \times \\
& \times \alpha_{2} \alpha_{3} \alpha_{4} G_{\alpha_{2} \alpha_{3}}^{\mathrm{c}(0)}(2 \mid 3) G_{\alpha_{4} \alpha^{\prime}}^{\mathrm{c}(0)}\left(4 \mid x^{\prime}\right) .
\end{aligned}
$$

Графически этот вклад представлен на рис. 2, где изображены диаграммы нулевого "а", второго - "б" и четвертого - "в", “г” порядков по $V$. На схеме “д" дано графическое изображение уравнения Дайсона. Схема "e" показывает простейшие вклады в массовый оператор.

Первые три слагаемых в (18) имеют вид цепочки и не содержат неприводимых функций Грина f-электронов. Присутствие корреляций f-электронов впервые проявляется в четвертом порядке по $V$ и отражено в структуре массового оператора $\Sigma^{c}$.

Диаграммы рис. 2 содержат внешние вершины с-электронов со своими индексами. Поэтому для внутренних вершин указаны только $\alpha$-индексы с-операторов; $\alpha$-индексы f-операторов, противоположные по знаку индексам с-операторов, не указаны, чтобы не перегружать рисунок.

На основании вышеприведенных результатов нетрудно видеть, что для полной функции Грина с-электронов имеет место следующее уравнение Дайсона:

$$
G_{\alpha \alpha^{\prime}}^{\mathrm{c}}\left(x \mid x^{\prime}\right)=G_{\alpha \alpha^{\prime}}^{\mathrm{c}(0)}\left(x \mid x^{\prime}\right)+\sum_{\alpha_{1} \alpha_{2}} \sum_{12} G_{\alpha \alpha_{1}}^{\mathrm{c}(0)}(x \mid 1) \alpha_{1} \Sigma_{\alpha_{1} \alpha_{2}}^{\mathrm{c}}(1 \mid 2) \alpha_{2} G_{\alpha_{2} \alpha^{\prime}}^{\mathrm{c}}\left(2 \mid x^{\prime}\right),
$$

где

$$
\begin{aligned}
\Sigma_{\alpha \alpha^{\prime}}^{\mathrm{c}}\left(x \mid x^{\prime}\right)= & V^{2}\left[G_{\alpha \alpha^{\prime}}^{\mathrm{f}(0)}\left(x \mid x^{\prime}\right)-\frac{V^{2}}{2} \sum_{\alpha_{1} \alpha_{2}} \sum_{12} G_{\alpha_{2} \alpha_{1}}^{\mathrm{c}(0)}\left(x_{2} \mid x_{1}\right) \times\right. \\
& \left.\times \alpha_{1} \alpha_{2}\left\langle T f^{\alpha}(x) f^{\alpha_{1}}\left(x_{1}\right) f^{-\alpha_{2}}\left(x_{2}\right) f^{-\alpha^{\prime}}\left(x^{\prime}\right)\right\rangle_{0}^{\mathrm{ir}}+\cdots\right] .
\end{aligned}
$$




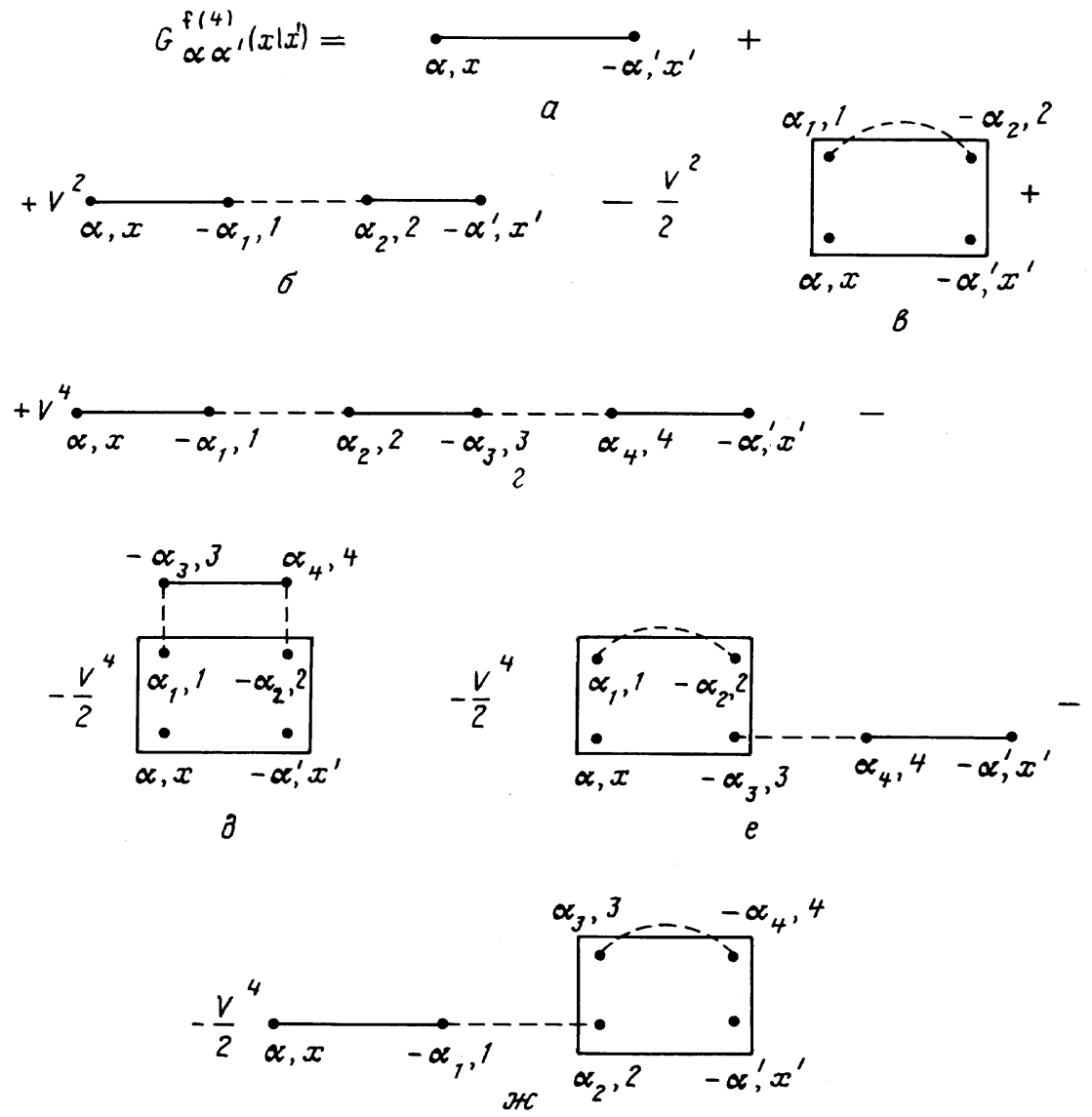

Рис. 3

По своему виду уравнение (19) характерно для теории слабой связи, отличаясь тем, что массовый оператор $\Sigma^{c}$ содержит вклады, обусловленные существованием сильных корреляций f-электронов и представленные в (20) простейшим образом. Более полно наличие этих вкладов может быть выражено следующим образом:

$$
\begin{aligned}
& \Sigma_{\alpha \alpha^{\prime}}^{\mathrm{c}}\left(x \mid x^{\prime}\right)=V^{2} \Lambda_{\alpha \alpha^{\prime}}\left(x \mid x^{\prime}\right), \\
& \Lambda_{\alpha \alpha^{\prime}}\left(x \mid x^{\prime}\right)=G_{\alpha \alpha^{\prime}}^{\mathrm{f}(0)}\left(x \mid x^{\prime}\right)+Z_{\alpha \alpha^{\prime}}\left(x \mid x^{\prime}\right) .
\end{aligned}
$$

Здесь функция $Z_{\alpha \alpha^{\prime}}$ является сильно связной неприводимой функцией, которая содержит все вклады от неприводимых функций Грина f-электронов таких, которые не могут быть разделены на части разрывом одной с-линии.

Графически уравнение (19) представлено на рис. 2д. При этом двойная штриховая линия изображает полную одночастичную функцию Грина с-электронов.

Как видно из вышеприведенных выражений всем внутренним вершинам сопоставляется (в соответствии с выражением (9)) дополнительный множитель $\alpha$. 


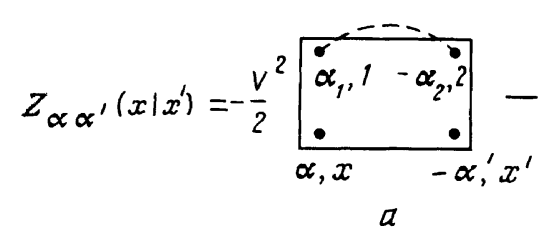
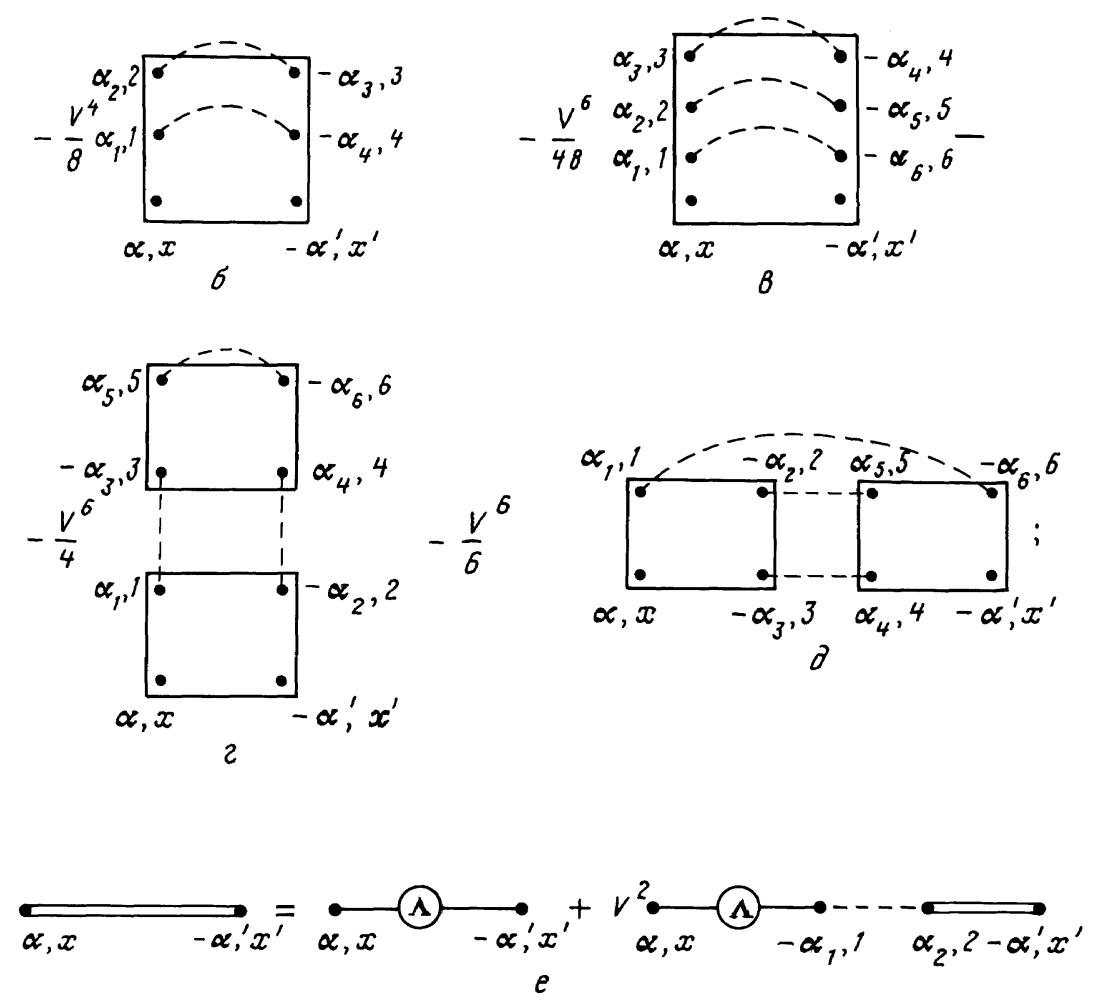

Рис. 4

Перейдем сейчас к f-подсистеме. Простейшие вклады для полной функции Грина f-электронов имеют вид

$$
\begin{gathered}
G_{\alpha \alpha^{\prime}}^{\mathrm{f}(2)}\left(x \mid x^{\prime}\right)=G_{\alpha \alpha^{\prime}}^{\mathrm{f}(0)}\left(x \mid x^{\prime}\right)+V^{2} \sum_{\alpha_{1} \alpha_{2}} \sum_{12} G_{\alpha \alpha_{1}}^{\mathrm{f}(0)}(x \mid 1) \alpha_{1} G_{\alpha_{1} \alpha_{2}}^{\mathrm{c}(0)}(1 \mid 2) \alpha_{2} G_{\alpha_{2} \alpha^{\prime}}^{\mathrm{f}(0)}\left(2 \mid x^{\prime}\right)- \\
-\frac{V^{2}}{2} \sum_{\alpha_{1} \alpha_{2}} \sum_{12}\left\langle T f^{\alpha}(x) f^{\alpha_{1}}(1) f^{-\alpha_{2}}(2) f^{-\alpha^{\prime}}\left(x^{\prime}\right)\right\rangle_{0}^{\mathrm{ir}} \alpha_{1} \alpha_{2} G_{\alpha_{2} \alpha_{1}}^{\mathrm{c}(0)}(2 \mid 1)
\end{gathered}
$$

Некоторые дополнительные вклады приведены на рис. 3. Диаграмма "а" отвечает нулевой функции Грина, "б" и "Г" - цепочечные диаграммы второго и четвертого порядков, диаграммы “в”, “д”, “е” и “ж” содержат простейшую неприводимую функцию Грина. 
На рис. 3 внешние вершины, отмеченные индексами $\alpha x$ и $-\alpha^{\prime} x^{\prime}$, принадлежат f-электронам. Поэтому для внутренних вершин, в которых сходятся линии как f-, так и с-электронов, указаны для простоты только $\alpha$-числа локализованных электронов; $\alpha$-индексы с-линий, соединяюших эти вершины, имеют противоположный указанному на рисунке знаку.

Диаграммы рис. 3в и 3 д являются сильно связными и дают вклад в функцию $Z_{\alpha \alpha^{\prime}}$. Диаграммы “е” и “ж” слабо связные и по определению в $Z_{\alpha \alpha}$ ' не входят.

На рис. 4 приведены примеры более сложных вкладов в функцию $Z_{\alpha \alpha^{\prime}}$ (диаграммы “а"-“д"). В диаграммах "а"-“г” внешние вершины $x$ и $x^{\prime}$ содержатся в одном и том же графическом элементе неприводимости, и поэтому эти вклады локальны. Они характеризуются узельным символом Кронекера $\delta \vec{x}, \vec{x}^{\prime}$ и независимостью от импульса соответствуюшего фурье-образа. Диаграмма "д” демонстрирует появление пространственной дисперсии корреляционной функции.

Отметим, что знаки диаграмм сушественно зависят от порядка расположения аргументов всех как одночастичных, так и многочастичных функций Грина. Диаграммы рис. $4 б$ и 4в демонстрируют замыкание на один прямоугольный элемент диаграммы нескольких пропагаторов с-электронов, что делает громоздким использование спинорного формализма Горькова-Намбу для записи их вкладов. Именно стремление упростить запись многовершинных вкладов неприводимой функции Грина и избежать многократных вычислений следов матриц, характерных для спинорного формализма, явилось причиной введения зарядовых чисел $\alpha$.

В качестве иллюстрации использования нового формализма приведем вклады трех диаграмм рис. 4 ;

$$
\begin{aligned}
& Z_{\alpha \alpha^{\prime}}^{\text {“б” }}\left(x \mid x^{\prime}\right)=-\frac{V^{4}}{8} \sum_{\alpha_{1}, \ldots, \alpha_{4}} \sum_{1, \ldots, 4}\left\langle T f^{\alpha}(x) f^{\alpha_{1}}(1) f^{\alpha_{2}}(2) f^{-\alpha_{3}}(3) f^{-\alpha_{4}}(4) f^{-\alpha^{\prime}}\left(x^{\prime}\right)\right\rangle_{0}^{\text {ir }} \times \\
& \times G_{\alpha_{3} \alpha_{2}}^{\mathrm{c}(0)}(3 \mid 2) G_{\alpha_{4} \alpha_{1}}^{\mathrm{c}(0)}(4 \mid 1) \alpha_{1} \alpha_{2} \alpha_{3} \alpha_{4}, \\
& Z_{\alpha \alpha^{\prime}}^{\text {"Г" }}\left(x \mid x^{\prime}\right)=-\frac{V^{6}}{4} \sum_{\alpha_{1}, \ldots, \alpha_{6}} \sum_{1, \ldots, 6}\left\langle T f^{\alpha}(x) f^{\alpha_{1}}(1) f^{-\alpha_{2}}(2) f^{-\alpha^{\prime}}\left(x^{\prime}\right)\right\rangle_{0}^{\mathrm{ir}} \times \\
& \times\left\langle T f^{-\alpha_{3}}(3) f^{\alpha_{4}}(4) f^{\alpha_{5}}(5) f^{-\alpha_{6}}(6)\right\rangle_{0}^{\mathrm{ir}} G_{\alpha_{2} \alpha_{4}}^{\mathrm{c}(0)}(2 \mid 4) G_{\alpha_{3} \alpha_{1}}^{\mathrm{c}(0)}(3 \mid 1) G_{\alpha_{6} \alpha_{5}}^{\mathrm{c}(0)}(6 \mid 5) \times \\
& \times \alpha_{1} \alpha_{2} \alpha_{3} \alpha_{4} \alpha_{5} \alpha_{6}
\end{aligned}
$$

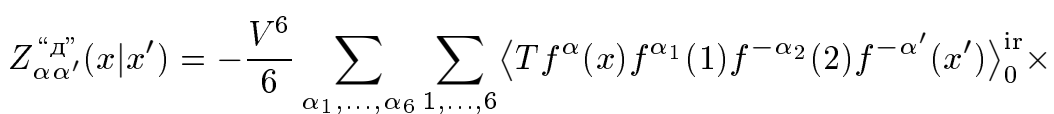

$$
\begin{aligned}
& \times\left\langle T f^{\alpha_{4}}(4) f^{\alpha_{5}}(5) f^{-\alpha_{6}}(6) f^{-\alpha^{\prime}}\left(x^{\prime}\right)\right\rangle_{0}^{\mathrm{ir}} G_{\alpha_{2} \alpha_{5}}^{\mathrm{c}(0)}(2 \mid 5) G_{\alpha_{3} \alpha_{4}}^{\mathrm{c}(0)}(3 \mid 4) G_{\alpha_{6} \alpha_{1}}^{\mathrm{c}(0)}(6 \mid 1) \times \\
& \times \alpha_{1} \alpha_{2} \alpha_{3} \alpha_{4} \alpha_{5} \alpha_{6} \text {. }
\end{aligned}
$$

Корреляционная функция $Z_{\alpha \alpha^{\prime}}$, содержашая основные зарядовые магнитные и сверхпроводящие флуктуации системы, является главным элементом уравнения Дайсона f-электронов. В теории систем со слабым взаимодействием таким основным элементом является массовый оператор системы. В нашем случае роль массового оператора выполняет свободный пропагатор с-электронов, умноженный на $V^{2}$. 
На основании структуры диаграмм рис. 3 и 4 нетрудно написать уравнение Дайсона для f-электронов

$$
\begin{aligned}
G_{\alpha \alpha^{\prime}}^{\mathrm{f}}\left(x \mid x^{\prime}\right)= & \Lambda_{\alpha \alpha^{\prime}}\left(x \mid x^{\prime}\right)+V^{2} \sum_{\alpha_{1} \alpha_{2}} \sum_{12} \Lambda_{\alpha \alpha_{1}}(x \mid 1) \alpha_{1} \times \\
& \times G_{\alpha_{1} \alpha_{2}}^{\mathrm{c}(0)}(1 \mid 2) \alpha_{2} G_{\alpha_{2} \alpha^{\prime}}^{\mathrm{f}}\left(2 \mid x^{\prime}\right) .
\end{aligned}
$$

Графическое изображение этого уравнения приведено на рис. 4е. Величина $\Lambda_{\alpha \alpha^{\prime}}$, содержащая сумму свободного пропагатора и корреляционной функции $Z_{\alpha \alpha^{\prime}}$, определена формулой (21). В уравнении (26) так же, как и в предыдуших выражениях, подразумевается суммирование по дискретным и интегрирование по непрерывным индексам 1 и 2.

Анализ вкладов диаграмм для смешанных функций Грина с- и f-электронов позволяет установить следуюшие соотношения между этими последними и полной функцией f-электронов:

$$
\begin{aligned}
& G_{\alpha \alpha^{\prime}}^{\mathrm{cf}}\left(x \mid x^{\prime}\right)=V \sum_{\alpha_{1} 1} G_{\alpha \alpha_{1}}^{\mathrm{c}(0)}(x \mid 1) \alpha_{1} G_{\alpha_{1} \alpha^{\prime}}^{\mathrm{f}}\left(1 \mid x^{\prime}\right), \\
& G_{\alpha \alpha^{\prime}}^{\mathrm{fc}}\left(x \mid x^{\prime}\right)=V \sum_{\alpha_{1} 1} G_{\alpha \alpha_{1}}^{\mathrm{f}}(x \mid 1) \alpha_{1} G_{\alpha_{1} \alpha^{\prime}}^{\mathrm{c}(0)}\left(1 \mid x^{\prime}\right) .
\end{aligned}
$$

В уравнениях (19), (26) и (27) для фермиевских функций Грина легко перейти к фурье-представлению вследствие их пространственной и временной однородности с помощью следующего определения фурье-образов:

$$
f(\vec{x}, \tau)=\frac{1}{N} \sum_{\vec{k}} \frac{1}{\beta} \sum_{\omega_{n}} f\left(\vec{k} \mid i \omega_{n}\right) e^{-i \vec{k} \vec{x}-i \omega_{n} \tau} .
$$

После этого перехода останутся невыполненными суммирования по дискретным спиновым и зарядовым индексам $(\sigma, \alpha)$. При этом суммирования по $\alpha$ перебирают автоматически все нормальные и аномальные функции Грина, тогда как спиновое суммирование нуждается для своего осушествления в определенных предположениях относительно магнитного состояния системы и характера сверхпроводящего связывания электронов путем образования синглетов или триплетов.

\section{3. СИНГЛЕТНАЯ И ТРИПЛЕТНАЯ СВЕРХПРОВОДИМОСТЬ}

Перепишем основные уравнения теории (19), (26) и (27) в фурье-компонентах $(k=$ $\left.\vec{k}, i \omega_{n}\right)$ :

$$
\begin{aligned}
& G_{\alpha \alpha^{\prime}}^{\mathrm{c}}\left(\sigma, \sigma^{\prime} \mid k\right)=\delta_{\sigma, \sigma^{\prime}} \delta_{\alpha, \alpha^{\prime}} G_{\alpha \alpha}^{\mathrm{c}(0)}(\sigma \mid k)+ \\
& +\alpha G_{\alpha \alpha}^{\mathrm{c}(0)}(\sigma \mid k) \sum_{\alpha_{1} \sigma_{1}} \Sigma_{\alpha \alpha_{1}}^{\mathrm{c}}\left(\sigma, \sigma_{1} \mid k\right) \alpha_{1} G_{\alpha_{1} \alpha^{\prime}}^{\mathrm{c}}\left(\sigma_{1}, \sigma^{\prime} \mid k\right), \\
& G_{\alpha \alpha^{\prime}}^{\mathrm{f}}\left(\sigma, \sigma^{\prime} \mid k\right)=\Lambda_{\alpha \alpha^{\prime}}\left(\sigma, \sigma^{\prime} \mid k\right)+ \\
& +V^{2} \sum_{\alpha_{1} \sigma_{1}} \Lambda_{\alpha \alpha_{1}}\left(\sigma, \sigma_{1} \mid k\right) G_{\alpha_{1} \alpha_{1}}^{\mathrm{c}(0)}\left(\sigma_{1} \mid k\right) G_{\alpha_{1} \alpha^{\prime}}^{\mathrm{f}}\left(\sigma_{1}, \sigma^{\prime} \mid k\right), \\
& \left\{\begin{array}{l}
G_{\alpha \alpha^{\prime}}^{\mathrm{cf}}\left(\sigma, \sigma^{\prime} \mid k\right)=\alpha V G_{\alpha \alpha}^{\mathrm{c}(0)}(\sigma \mid k) G_{\alpha \alpha^{\prime}}^{\mathrm{f}}\left(\sigma, \sigma^{\prime} \mid k\right), \\
G_{\alpha \alpha^{\prime}}^{\mathrm{f}}\left(\sigma, \sigma^{\prime} \mid k\right)=\alpha^{\prime} V G_{\alpha \alpha^{\prime}}^{\mathrm{f}}\left(\sigma, \sigma^{\prime} \mid k\right) G_{\alpha^{\prime} \alpha^{\prime}}^{\mathrm{c}(0)}\left(\sigma^{\prime} \mid k\right) .
\end{array}\right.
\end{aligned}
$$


Здесь использовано свойство диагональности как по зарядовым, так и по спиновым индексам нулевого пропагатора с-электронов. Фурье-компоненты этих функций обладают следующими свойствами симметрии:

$$
\begin{aligned}
& G_{\alpha \alpha^{\prime}}^{\mathrm{a}}\left(\sigma, \sigma^{\prime} \mid k\right)=-G_{-\alpha^{\prime},-\alpha}^{\mathrm{a}}\left(\sigma^{\prime}, \sigma \mid-k\right), \\
& G_{\alpha \alpha^{\prime}}^{\mathrm{cf}}\left(\sigma, \sigma^{\prime} \mid k\right)=-G_{-\alpha^{\prime},-\alpha}^{\mathrm{f}}\left(\sigma^{\prime}, \sigma \mid-k\right), \quad \mathrm{a}=\mathrm{c}, \mathrm{f} .
\end{aligned}
$$

Зададим теперь в уравнениях (29)-(31) зарядовые числа $\alpha, \alpha^{\prime}$, выполним суммирование по $\alpha_{1}$ и перейдем на основании соотношений (11) к нормальным и аномальным функциям Грина, зависящим от четырехмерного импульса $k=\left(\vec{k}, i \omega_{n}\right)$ и спиновых индексов $\sigma, \sigma^{\prime}$. Введем специальные обозначения для диагональных и недиагональных корреляционных функций f-электронов:

$$
\begin{aligned}
Z_{1,1}\left[\sigma, \sigma^{\prime} \mid k\right] & =Z_{\sigma \sigma^{\prime}}(k), \\
Z_{1,-1}\left[\sigma, \sigma^{\prime} \mid k\right] & =Y_{\sigma \sigma^{\prime}}(k), \\
Z_{-1,1}\left[\sigma, \sigma^{\prime} \mid k\right] & =\bar{Y}_{\sigma \sigma^{\prime}}(k), \\
Z_{-1,-1}\left[\sigma, \sigma^{\prime} \mid k\right] & =-Z_{\sigma^{\prime} \sigma}(-k) .
\end{aligned}
$$

Учитывая эти обозначения и определения (21), получим вместо уравнения (29) следуюшую систему уравнений:

$$
\begin{aligned}
& G_{\sigma \sigma^{\prime}}^{\mathrm{c}}(k)=\delta_{\sigma, \sigma^{\prime}} G_{\sigma}^{\mathrm{c}(0)}(k)+G_{\sigma}^{\mathrm{c}(0)}(k) V^{2} \sum_{\sigma_{1}}\left[\Lambda_{\sigma \sigma_{1}}(k) G_{\sigma_{1} \sigma^{\prime}}^{\mathrm{c}}(k)-Y_{\sigma \sigma_{1}}(k) \bar{F}_{\sigma_{1} \sigma^{\prime}}^{\mathrm{c}}(k)\right], \\
& F_{\sigma \sigma^{\prime}}^{\mathrm{c}}(k)=G_{\sigma}^{\mathrm{c}(0)}(k) V^{2} \sum_{\sigma_{1}}\left[\Lambda_{\sigma \sigma_{1}}(k) F_{\sigma_{1} \sigma^{\prime}}^{\mathrm{c}}(k)+Y_{\sigma \sigma_{1}}(k) G_{\sigma^{\prime} \sigma_{1}}^{\mathrm{c}}(-k)\right], \\
& \bar{F}_{\sigma \sigma^{\prime}}^{\mathrm{c}}(k)=G_{\sigma}^{\mathrm{c}(0)}(-k) V^{2} \sum_{\sigma_{1}}\left[\bar{Y}_{\sigma \sigma_{1}}(k) G_{\sigma_{1} \sigma^{\prime}}^{\mathrm{c}}(k)+\Lambda_{\sigma_{1} \sigma}(-k) \bar{F}_{\sigma_{1} \sigma^{\prime}}^{\mathrm{c}}(k)\right] .
\end{aligned}
$$

На основании уравнения (30) можно получить аналогичную систему уравнений для f-электронов

$$
\begin{aligned}
G_{\sigma \sigma^{\prime}}^{\mathrm{f}}(k)= & \Lambda_{\sigma \sigma^{\prime}}(k)+V^{2} \sum_{\sigma_{1}}\left[\Lambda_{\sigma \sigma_{1}}(k) G_{\sigma_{1}}^{\mathrm{c}(0)}(k) G_{\sigma_{1} \sigma^{\prime}}^{\mathrm{f}}(k)-Y_{\sigma \sigma_{1}}(k) G_{\sigma_{1}}^{\mathrm{c}(0)}(-k) \bar{F}_{\sigma_{1} \sigma^{\prime}}^{\mathrm{f}}(k)\right], \\
F_{\sigma \sigma^{\prime}}^{\mathrm{f}}(k)= & Y_{\sigma \sigma^{\prime}}(k)+V^{2} \sum_{\sigma_{1}}\left[\Lambda_{\sigma \sigma_{1}}(k) G_{\sigma_{1}}^{\mathrm{c}(0)}(k) F_{\sigma_{1} \sigma^{\prime}}^{\mathrm{f}}(k)+Y_{\sigma \sigma_{1}}(k) G_{\sigma_{1}}^{\mathrm{c}(0)}(-k) G_{\sigma^{\prime} \sigma_{1}}^{\mathrm{f}}(-k)\right], \\
\bar{F}_{\sigma \sigma^{\prime}}^{\mathrm{f}}(k)= & \bar{Y}_{\sigma \sigma^{\prime}}(k)+V^{2} \sum_{\sigma_{1}}\left[\bar{Y}_{\sigma \sigma_{1}}(k) G_{\sigma_{1}}^{\mathrm{c}(0)}(k) G_{\sigma_{1} \sigma^{\prime}}^{\mathrm{f}}(k)+\right. \\
& \left.+\Lambda_{\sigma_{1} \sigma}(-k) G_{\sigma_{1}}^{\mathrm{c}(0)}(-k) \bar{F}_{\sigma_{1} \sigma^{\prime}}^{\mathrm{f}}(k)\right] .
\end{aligned}
$$

И, наконец, на основании уравнений (31), нетрудно установить равенства

$$
\begin{aligned}
G_{\sigma \sigma^{\prime}}^{\mathrm{cf}}(k) & =V G_{\sigma}^{\mathrm{c}(0)}(k) G_{\sigma \sigma^{\prime}}^{\mathrm{f}}(k), \\
F_{\sigma \sigma^{\prime}}^{\mathrm{cf}}(k) & =V G_{\sigma}^{\mathrm{c}(0)}(k) F_{\sigma \sigma^{\prime}}^{\mathrm{f}}(k), \\
\bar{F}_{\sigma \sigma^{\prime}}^{\mathrm{cf}}(k) & =V G_{\sigma}^{\mathrm{c}(0)}(-k) \bar{F}_{\sigma \sigma^{\prime}}^{\mathrm{f}}(k) .
\end{aligned}
$$


Уравнения (34) и (35) допускают описание различных сверхпроводящих фаз на фоне различных магнитных состояний системы.

Рассмотрим реализацию синглетной сверхпроводимости на фоне парамагнитного состояния вещества. В этом случае имеется следующая спиновая зависимость функций Грина $(\mathrm{a}=\mathrm{c}, \mathrm{f})$ :

$$
\begin{aligned}
G_{\sigma \sigma^{\prime}}^{\mathrm{a}}(k) & =\delta_{\sigma, \sigma^{\prime}} G_{\sigma}^{\mathrm{a}}(k), \\
F_{\sigma \sigma^{\prime}}^{\mathrm{a}}(k) & =\delta_{\sigma^{\prime},-\sigma} F_{\sigma,-\sigma}^{\mathrm{a}}(k), \\
\bar{F}_{\sigma^{\prime} \sigma}^{\mathrm{a}}(k) & =\delta_{\sigma^{\prime},-\sigma} \bar{F}_{-\sigma, \sigma}^{\mathrm{a}}(k)
\end{aligned}
$$

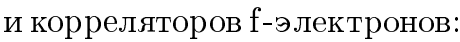

$$
\begin{aligned}
& \Lambda_{\sigma \sigma^{\prime}}(k)=\delta_{\sigma^{\prime}, \sigma} \Lambda_{\sigma}(k), \\
& Y_{\sigma \sigma^{\prime}}(k)=\delta_{\sigma^{\prime},-\sigma} Y_{\sigma,-\sigma}(k), \\
& \bar{Y}_{\sigma^{\prime} \sigma}(k)=\delta_{\sigma^{\prime},-\sigma} \bar{Y}_{-\sigma, \sigma}(k) .
\end{aligned}
$$

Уравнения (34) с учетом соотношений (37) и (38) принимают вид

$$
\begin{aligned}
G_{\sigma}^{\mathrm{c}}(k) & =G_{\sigma}^{\mathrm{c}(0)}(k)+G_{\sigma}^{\mathrm{c}(0)}(k) V^{2}\left[\Lambda_{\sigma}(k) G_{\sigma}^{\mathrm{c}}(k)-Y_{\sigma,-\sigma}(k) \bar{F}_{-\sigma, \sigma}^{\mathrm{c}}(k)\right], \\
F_{\sigma,-\sigma}^{\mathrm{c}}(k) & =G_{\sigma}^{\mathrm{c}(0)}(k) V^{2}\left[\Lambda_{\sigma}(k) F_{\sigma,-\sigma}^{\mathrm{c}}(k)+Y_{\sigma,-\sigma}(k) G_{-\sigma}^{\mathrm{c}}(-k)\right], \\
\bar{F}_{-\sigma, \sigma}^{\mathrm{c}}(k) & =G_{\sigma}^{\mathrm{c}(0)}(-k) V^{2}\left[\bar{Y}_{-\sigma, \sigma}(k) G_{\sigma}^{\mathrm{c}}(k)+\Lambda_{-\sigma}(-k) \bar{F}_{-\sigma, \sigma}^{\mathrm{c}}(k)\right] .
\end{aligned}
$$

Эту систему уравнений можно переписать в спинорном представлении Горькова-Намбу в следующей форме:

$$
\widehat{G}_{\sigma}^{\mathrm{c}}(k)=\widehat{G}_{\sigma}^{\mathrm{c}(0)}(k)+V^{2} \widehat{G}_{\sigma}^{\mathrm{c}(0)}(k) \hat{\sigma}_{3} \hat{\Lambda}_{\sigma}(k) \hat{\sigma}_{3} \widehat{G}_{\sigma}^{\mathrm{c}}(k)
$$

где введены спинорные матрищы $(\mathrm{a}=\mathrm{c}, \mathrm{f})$

$$
\begin{aligned}
& \widehat{G}_{\sigma}^{\mathrm{a}}(k)=\left(\begin{array}{cc}
G_{\sigma}^{\mathrm{a}}(k) & F_{\sigma,-\sigma}^{\mathrm{a}}(k) \\
\bar{F}_{-\sigma, \sigma}^{\mathrm{a}}(k) & -G_{-\sigma}^{\mathrm{a}}(-k)
\end{array}\right), \\
& \widehat{\Lambda}_{\sigma}(k)=\left(\begin{array}{cc}
\Lambda_{\sigma}(k) & Y_{\sigma,-\sigma}(k) \\
\bar{Y}_{-\sigma, \sigma}(k) & -\Lambda_{-\sigma}(-k)
\end{array}\right),
\end{aligned}
$$

а $\hat{\sigma}$ - матрицы Паули.

В окончательном виде уравнения Дайсона для с-электронных функций Грина в синглетном состоянии имеют вид

$$
\begin{aligned}
G_{\sigma}^{\mathrm{c}}(k)= & G_{\sigma}^{\mathrm{c}(0)}(k)\left[1-V^{2} G_{-\sigma}^{\mathrm{c}(0)}(-k) \Lambda_{-\sigma}(-k)\right] D_{\sigma}(k)^{-1}, \\
\bar{F}_{-\sigma, \sigma}^{\mathrm{c}}(k)= & V^{2} G_{-\sigma}^{\mathrm{c}(0)}(-k) G_{\sigma}^{\mathrm{c}(0)}(k) \bar{Y}_{-\sigma, \sigma}(k) D_{\sigma}^{-1}(k), \\
F_{\sigma,-\sigma}^{\mathrm{c}}(k)= & V^{2} G_{-\sigma}^{\mathrm{c}(0)}(-k) G_{\sigma}^{\mathrm{c}(0)}(k) Y_{\sigma,-\sigma}(k) D_{\sigma}^{-1}(k), \\
D_{\sigma}(k)= & {\left[1-V^{2} G_{-\sigma}^{\mathrm{c}(0)}(-k) \Lambda_{-\sigma}(-k)\right]\left[1-V^{2} G_{\sigma}^{\mathrm{c}(0)}(k) \Lambda_{\sigma}(k)\right]+} \\
& +V^{4} G_{\sigma}^{\mathrm{c}(0)}(k) G_{-\sigma}^{\mathrm{c}(0)}(-k) Y_{\sigma,-\sigma}(k) \bar{Y}_{-\sigma, \sigma}(k) .
\end{aligned}
$$


Для f-электронов также может быть написано соответствующее спинорное уравнение Дайсона с решениями

$$
\begin{aligned}
G_{\sigma}^{\mathrm{f}}(k)= & \left\{\Lambda_{\sigma}(k)\left[1-V^{2} G_{-\sigma}^{\mathrm{c}(0)}(-k) \Lambda_{-\sigma}(-k)\right]-\right. \\
& \left.-V^{2} G_{-\sigma}^{\mathrm{c}(0)}(-k) \bar{Y}_{-\sigma, \sigma}(k) Y_{\sigma,-\sigma}(k)\right\} D_{\sigma}^{-1}(k), \\
F_{\sigma,-\sigma}^{\mathrm{f}}(k)= & Y_{\sigma,-\sigma}(k) D_{\sigma}(k)^{-1}, \\
\bar{F}_{-\sigma, \sigma}^{\mathrm{f}}(k)= & \bar{Y}_{-\sigma, \sigma}(k) D_{\sigma}^{-1}(k) .
\end{aligned}
$$

Существенно отметить следующие свойства симметрии параметров порядка сверхпроводяшей фазы $Y, \bar{Y}$ и аномальных функций Грина $(\mathrm{a}=\mathrm{c}, \mathrm{f})$ :

$$
\begin{aligned}
Y_{\sigma,-\sigma}(k) & =-Y_{-\sigma, \sigma}(-k), & \bar{Y}_{-\sigma, \sigma}(k) & =-\bar{Y}_{\sigma,-\sigma}(-k), \\
F_{\sigma,-\sigma}^{\mathrm{a}}(k) & =-F_{-\sigma, \sigma}^{\mathrm{a}}(-k), & \bar{F}_{-\sigma, \sigma}^{\mathrm{a}}(k) & =-\bar{F}_{\sigma,-\sigma}^{\mathrm{a}}(-k), \\
D_{\sigma}(k) & =D_{-\sigma}(-k) . & &
\end{aligned}
$$

Рассмотрим теперь возможность реализации триплетной сверхпроводимости на фоне парамагнитной фазы. Спиновая зависимость параметров порядка и аномальных функций Грина в этом случае является диагональный $(\mathrm{a}=\mathrm{c}, \mathrm{f})$, т.е.

$$
\begin{aligned}
& G_{\sigma \sigma^{\prime}}^{\mathrm{a}}(k)=\delta_{\sigma^{\prime}, \sigma} G_{\sigma}^{\mathrm{a}}(k), \quad F_{\sigma \sigma^{\prime}}^{\mathrm{a}}(k)=\delta_{\sigma^{\prime}, \sigma} F_{\sigma}^{\mathrm{a}}(k), \\
& \bar{F}_{\sigma \sigma^{\prime}}^{\mathrm{a}}(k)=\delta_{\sigma^{\prime}, \sigma} \bar{F}_{\sigma}^{\mathrm{a}}(k), \quad \Lambda_{\sigma \sigma^{\prime}}(k)=\delta_{\sigma^{\prime}, \sigma} \Lambda_{\sigma}(k) \text {, } \\
& Y_{\sigma \sigma^{\prime}}(k)=\delta_{\sigma^{\prime}, \sigma} Y_{\sigma}(k), \quad \bar{Y}_{\sigma \sigma^{\prime}}(k)=\delta_{\sigma^{\prime}, \sigma} \bar{Y}_{\sigma}(k) \text {. }
\end{aligned}
$$

Выполняются свойства симметрии

$$
\begin{array}{ll}
F_{\sigma}^{\mathrm{a}}(k)=-F_{\sigma}^{\mathrm{a}}(-k), & \bar{F}_{\sigma}^{\mathrm{a}}(k)=-\bar{F}_{\sigma}^{\mathrm{a}}(-k), \\
Y_{\sigma}(k)=-Y_{\sigma}(-k), & \bar{Y}_{\sigma}(k)=-\bar{Y}_{\sigma}(-k) .
\end{array}
$$

При этих предположениях о спиновой зависимости динамических величин и на основании уравнений (34) и (35) для триплетной сверхпроводимости получаем уравнения Дайсона. Для с-электронов они имеют вид

$$
\begin{aligned}
G_{\sigma}^{\mathrm{c}}(k)= & G_{\sigma}^{\mathrm{c}(0)}(k)\left[1-V^{2} G_{\sigma}^{\mathrm{c}(0)}(-k) \Lambda_{\sigma}(-k)\right] D_{\sigma}^{\mathrm{t}}(k)^{-1}, \\
F_{\sigma}^{\mathrm{c}}(k)= & V^{2} G_{\sigma}^{\mathrm{c}(0)}(k) G_{\sigma}^{\mathrm{c}(0)}(-k) Y_{\sigma}(k) D_{\sigma}^{\mathrm{t}}(k)^{-1}, \\
\bar{F}_{\sigma}^{\mathrm{c}}(k)= & V^{2} G_{\sigma}^{\mathrm{c}(0)}(k) G_{\sigma}^{\mathrm{c}(0)}(-k) \bar{Y}_{\sigma}(k) D_{\sigma}^{\mathrm{t}}(k)^{-1}, \\
D_{\sigma}^{\mathrm{t}}(k)= & {\left[1-V^{2} G_{\sigma}^{\mathrm{c}(0)}(k) \Lambda_{\sigma}(k)\right]\left[1-G_{\sigma}^{\mathrm{c}(0)}(-k) V^{2} \Lambda_{\sigma}(-k)\right]+} \\
& +V^{4} G_{\sigma}^{\mathrm{c}(0)}(k) G_{\sigma}^{\mathrm{c}(0)}(-k) Y_{\sigma}(k) \bar{Y}_{\sigma}(k) .
\end{aligned}
$$


Для f-подсистемы имеем

$$
\begin{aligned}
G_{\sigma}^{\mathrm{f}}(k)= & \left\{\Lambda_{\sigma}(k)\left[1-V^{2} G_{\sigma}^{\mathrm{c}(0)}(-k) \Lambda_{\sigma}(-k)\right]-\right. \\
& \left.-V^{2} G_{\sigma}^{\mathrm{c}(0)}(-k) Y_{\sigma}(k) \bar{Y}_{\sigma}(k)\right\} D_{\sigma}^{\mathrm{t}}(k)^{-1}, \\
F_{\sigma}^{\mathrm{f}}(k)= & Y_{\sigma}(k) D_{\sigma}^{\mathrm{t}}(k)^{-1} \\
\bar{F}_{\sigma}^{\mathrm{f}}(k)= & \bar{Y}_{\sigma}(k) D_{\sigma}^{\mathrm{t}}(k)^{-1} .
\end{aligned}
$$

Заданием полного числа с- и $\mathrm{f}$-электронов $N_{\mathrm{e}}$ определяется значение химического потенциала системы

$$
\sum_{\vec{k}} \frac{1}{\beta} \sum_{\omega_{n}} \sum_{\sigma} e^{i \omega_{n} 0^{+}}\left[G_{\sigma}^{\mathrm{c}}\left(\vec{k} \mid i \omega_{n}\right)+G_{\sigma}^{\mathrm{f}}\left(\vec{k} \mid i \omega_{n}\right)\right]=N_{\mathrm{e}}
$$

Чтобы найти параметры порядка $Y_{\sigma \sigma^{\prime}}, \bar{Y}_{\sigma \sigma^{\prime}}$ сверхпроводящей фазы и корреляционную функцию $Z_{\sigma \sigma^{\prime}}$, определяюшую тот фон, на котором реализуется сверхпроводимость, следует выделить класс диаграмм, сушественных для описания рассматриваемого явления. Примером такого суммирования сушественных диаграмм для описания синглетной сверхпроводимости является аппроксимация, предложенная в работах $[8,9]$ для описания сверхпроводимости в модели Хаббарда. Как нетрудно видеть, имеется тесная аналогия между уравнениями работ $[8,9]$ и полученными в данной работе определениями (43).

\section{4. ЗАКЛЮЧЕНИЕ}

Развита термодинамическая теория возмущений по степеням матричного элемента гибридизации с- и f-состояний электронов для сверхпроводяшего состояния периодической модели Андерсона.

Предложена новая диаграммная техника, существенными элементами которой являются обобщенная теорема Вика и неприводимые функции Грина сильно коррелированных f-электронов. На ее основе исследованы свойства ренормированных одночастичных функций Грина с- и f-электронов сверхпроводящего состояния указанной модели. В результате введения нового зарядового квантового числа $\alpha$ получена обобщенная запись этих уравнений, допускаюшая как синглетное, так и триплетное связывание электронов обеих подсистем. Введение этого квантового числа позволяет на равноправной основе рассматривать нормальные и аномальные функции Грина любого числа частиц и иметь простую запись вкладов неприводимых функций Грина. Ценой такого упрошения является появление дополнительных множителей во внутренних вершинах диаграмм, а также дополнительных суммирований по этим числам для всех внутренних линий.

Переход от сверхпроводяшего к нормальному состоянию вешества происходит после наложения дополнительного условия обрашения в нуль суммы всех $\alpha$-чисел функций Грина.

Показано, что благодаря гибридизации сверхпроводящее состояние наступает одновременно в обеих подсистемах с едиными параметрами порядка этой фазы. 
На основании полученных общих уравнений установлены особенности синглетной и триплетной сверхпроводимости на фоне парамагнитной фазы системы.

Установлена аналогия между уравнениями самосогласования синглетной сверхпроводимости данной модели и уравнениями однозонной модели Хаббарда. Различие состоит в том, что величина зонной энергии $\epsilon(\vec{k})$, фигурируюшая в уравнениях модели Хаббарда, в рассматриваемой модели заменяется динамической величиной, равной произведению $V^{2} G_{\sigma}^{\mathrm{c}(0)}(k)$.

Хочу выразить благодарность профессору Н. М. Плакиде за поддержку и ценное обсуждение.

Работа выполнена в рамках проекта "Гейзенберг-Ландау".

\section{Список литературы}

[1] J. Hubbard. Proc. R. Soc. A. 1963. V. 276. P. 233.

[2] P. W. Anderson. Phys. Rev. 1961. V. 124. P. 41.

[3] М.И. Владимир, В.А. Москаленко. ТМФ. 1990. Т. 82. С. 428.

[4] С.И. Вакару, М.И. Владимир, В.А. Москаленко. ТМФ. 1990. Т. 85. С. 248.

[5] С. П. Кожскару, В. А. Москаленко. ТМФ. 1993. Т. 97. С. 270.

[6] V.A. Moskalenko, S.P. Cojocaru, M. I. Vladimir. A diagram technique for strongly interacting systems. One- and two-band Hubbard Models. Preprint ICTP, IC/94/182. Trieste, Italy, 1994.

[7] Н. Н. Боголюбов, В. А. Москаленко. ДАН СССР. 1991. Т. 316. № 5. С. 1107.

[8] Н.Н. Боголюбов, В. А. Москаленко. ТМФ. 1991. Т. 86. С. 16.

[9] Н. Н. Боголюбов, В. А. Москаленко. ТМФ. 1992. Т. 92. С. 182.

[10] V. A. Moskalenko, D. F. Digor, L. A. Dogotaru, I. G. Porcescu. J. Low Temper. Phys. 1996. V. 105. № 3/4. P. 633; J. Phys. Studies. 1997. V. 1. № 3. P. 453.

[11] В. А. Москаленко. ТМФ. 1997. Т. 110. С. 308.

[12] V.A. Moskalenko, D.F. Digor, M. I. Vladimir, L. A. Dogotaru, I. G. Porcescu. Physica C. 1997. V. 282-287. P. 1717.

[13] Н. Н. Боголюбов. Избранные труды. Т. III. Киев: Наукова Думка, 1971.

[14] А.А. Абрикосов, Л. П. Горьков, И. Е. Дзялоиинский. Методы квантовой теории поля в статистической физике. М.: Наука, 1962.

[15] П. М. Слободян, И. В. Стасюк. ТМФ. 1974. Т. 19. С. 423.

[16] Р. О. Зайцев. ЖЭТФ. 1975. Т. 68. С. 207; 1976. Т. 70. С. 1100.

[17] Ю. А. Изюмов, Ю. М. Скрябин. Статистическая механика магнитноупорядоченных систем. М.: Наука, 1987. 